\title{
Conocimiento del personal de enfermería sobre la intervención en crisis de primera instancia y Primeros Auxilios Psicológicos
}

\author{
Knowledge of the nursing staff about the intervention in crisis of first instance and Psychological \\ First Aid \\ Conhecimento da equipe de enfermagem sobre a intervenção em crise de primeira instância e \\ Primeiros Socorros Psicológicos do ensino médio
}

\author{
Rubén Carlos Tunqui Cruz \\ carlostunqui@gmail.com \\ https://orcid.org/0000-0002-7450-3765 \\ Universidad Mayor Real y Pontificia de San Francisco Javier de Chuquisaca
Potosí, Bolivia
}

Recibido: febrero 2021 Revisado: marzo 2021 Aceptado: abril 2021

\begin{abstract}
RESUMEN
La intervención en una crisis a través de la aplicación de los Primeros Auxilios Psicológicos permite prestar ayuda psicológica inmediata para recuperar el equilibrio emocional de la persona afectada por el evento estresor. En este artículo se determina el conocimiento que posee el personal de los Centros de salud del municipio de Potosí acerca delos Primeros Auxilios Psicológicos. La población fue de 418 profesionales de la enfermería que laboran en los centros de salud de primer, segundo y tercer nivel, la muestra quedó constituida por 171 profesionales de la enfermería y 6 directivos de los centros de salud. El diseño fue de campo, transversal y las técnicas de recolección de los datos fueron la encuesta y la entrevista; los instrumentos el cuestionario y el guion de entrevista. Como hallazgo de la investigación se encontró que el personal de salud no está capacitado para abordar las crisis psicológicas aplicando los Primeros Auxilios Psicológicos.
\end{abstract}

Palabras clave: Primeros Auxilios Psicológicos (PAPsi); Personal de enfermería; afrontamiento de crisis; crisis psicológica

\begin{abstract}
Intervention in a crisis through the application of Psychological First Aid allows immediate psychological help to regain the emotional balance of the person affected by the stressful event. This article determines the knowledge that the personnel of the Health Centers of the municipality of Potosí have about Psychological First Aid. The population consisted of 418 nursing professionals who work in first, second and third level health centers; the sample was made up of 171 nursing professionals and 6 health center managers. The design was field, cross-sectional and the data collection techniques were the survey and the interview; the instruments the questionnaire and the interview script. As a research finding, it was found that health personnel are not trained to address psychological crises by applying Psychological First Aid.
\end{abstract}

Key words:

Psychological First Aid (PAPsi); Nursing staff; crisis coping; psychological crisis
RESUMO

A intervenção em uma crise por meio da aplicação de Primeiros Socorros Psicológicos permite 0 auxílio psicológico imediato para recuperar - equilíbrio emocional da pessoa afetada pelo evento estressante. Este artigo determina o conhecimento que os profissionais dos Centros de Saúde do município de Potosí possuem sobre o Pronto Atendimento Psicológico. A população foi constituída por 418 profissionais de enfermagem que atuam em centros de saúde de primeiro, segundo e terceiro nível, a amostra foi composta por 171 profissionais de enfermagem e 6 gestores de centros de saúde. O delineamento foi de campo, transversal e as técnicas de coleta de dados foram o survey e a entrevista; os instrumentos o questionário e o roteiro de entrevista. Como resultado da pesquisa, descobriu-se que o pessoal de saúde não é treinado para lidar com crises psicológicas aplicando os primeiros socorros psicológicos.

Palavras-chave: Primeiros Socorros Psicológicos (PAPsi); Equipe de enfermagem; enfrentamento de crises; crise psicológica 


\section{INTRODUCCIÓN}

Los Primeros Auxilios Psicológicos (PAPsi) se definen como una técnica de ayuda humanitaria de apoyo a otro ser humano que está sufriendo una crisis, y puede necesitar ayuda para recuperar su equilibrio emocional y prevenir el desarrollo de secuelas psicológicas (OPS, 2002; Proyecto Esfera, 2011; Federación internacional de sociedades de la Cruz Roja y Media Luna, 2014 y Cortés y Figueroa, 2017). La Organización Mundial de la Salud - OMS (2012) considera que los objetivos de los PAPsi son ayudar a las personas para que acepten lo sucedido mediante la expresión de sus sentimientos, facilitándoles así la búsqueda de soluciones y del apoyo social necesario. Se busca que el individuo recupere el equilibrio disminuyendo, de esta manera las posibles consecuencias negativas.

Los PAPsi buscan ofrecer ayuda de manera práctica y no invasiva centrándose en las necesidades y preocupaciones inmediatas de las personas y atenderlas en la medida de lo posible; escuchar al afectado sin sobre estimular para que hable sobre el evento estresor; reconfortar a las personas y ayudar a la calma con técnicas de relajación. Parte importante de esta técnica es estar disponible para aquellos que necesiten apoyo y ayuda, de ninguna manera forzando $u$ obligando a aquellos que no la necesitan o que se hayan negado a recibirla (OMS, 2012).

La función de los PAPsi no es realizar psicoterapia, ya que estos pueden ser ejecutados por personal no especializado; que haya sido capacitado en los conocimientos y las técnicas básicas. Generalmente las personas que están en continuo contacto con otros pueden ofrecer este tipo de ayuda, como el personal de enfermería, médicos, bomberos, docentes, consejeros, entre otros.

El objetivo de los PAPsi es mitigar los conflictos emocionales que se generan a causa de situaciones traumáticas, a través de la implementación de métodos o teorías psicológicas que permitirán ejercer control sobre la situación y brindar soporte a las personas que se encuentran en un estado de vulnerabilidad y por tanto es necesaria la intervención inmediata. Los PAPsi proporcionan el apoyo para que las personas afectadas logren un correcto afrontamiento de la situación traumática (Méndez, 2016).

El personal de enfermería pueden tener en el diseño curricular de su formación profesional un curso de "Técnicas de afrontamiento" que les permite desarrollar competencias cognitivas y conductuales para atender la crisis como situaciones que exceden o desbordan los recursos de una persona (Beltrán, 2017). En especial, el personal en enfermería debe contar con las herramientas básicas para poder atender en PAPsi al paciente o familiar que por una crisis lo requiera, ya que en los contextos hospitalarios las personas suelen acudir por situaciones de emergencia y estos acontecimientos pueden causar desequilibrio emocional al presentar miedo, tristeza, angustia, llanto y dolor.

Al respecto Slaikeu (1996), OMS (2012) Hernández y Gutiérrez (2014) y Cortés y Figueroa (2017) recomiendan algunas pautas prácticas de actuación, que facilitan el "cómo hacer" de los trabajadores de la salud que laboran en situaciones de emergencia. Se tienen en cuenta los elementos individuales y colectivos que deben conocer para planificar e implementar acciones concretas de intervención, estas acciones son:

1. Estrategias de contacto psicológico: Establecer contacto observar y escuchar de forma activa, para generar un vínculo con la persona afectada. No todas las personas van a necesitar PAP, debe dejar claro que usted está dispuesto a la ayuda, si la persona no la acepta no puede forzarla. En esta acción la persona se debe sentir segura y buscar su estabilización.

2. Examen de las dimensiones del problema: Consiste en el análisis de la situación para 
comprender las causas y consecuencias y tener una visión objetiva de la situación. Se enfoca a tres áreas: presente, pasado inmediato y futuro inmediato. Preguntas como: ¿Qué sucedió? ¿Cómo te sientes? ¿Qué puedes hacer? Pasado inmediato: ¿Quién? ¿Dónde? ¿Cuándo? ¿Cómo? Futuro inmediato: Enfocado a cuáles son los eventuales riesgos para la persona y prepararse para las soluciones inmediatas.

3. Analizar las posibles soluciones: las soluciones deben de estar en relación con la persona que está recibiendo los PAPsi, es decir que la presencia o no de crisis, dependerá de los significados que el individuo atribuya tanto a las demandas o estresores o situación de crisis como a sus capacidades para el afrontamiento; pues ambos factores intervienen para lograr un funcionamiento balanceado. Puede ayudar la categorización y jerarquización de situaciones que se deben atender y la prioridad para cada una.
4. Ejecutar la acción concreta: Las acciones se pueden sugerir, puede ayudar a contactar a las personas u organismos que puedan formar parte de la solución. Es importante que la persona active sus propios recursos o los recursos que puedan brindarle sus redes de apoyo familiares, amistades o comunitarias.

Si la persona llega a presentar una descompensación severa como producto de la crisis se debe derivar a urgencias psiquiátricas.

Dar seguimiento: Puede contactar a la persona para conocer cómo evoluciona su situación y ofrecer su apoyo.

Como guía para las acciones al aplicar los PAPsi en la Tabla 1 se resumen algunas acciones que son convenientes para ejecutar y cuáles deben evitarse (Slaikeu, 1996 y National Child Traumatic Stress Network, sf).

Tabla 1. Acciones convenientes y no convenientes de ejecutar en la aplicación de los PAPsi.

\begin{tabular}{|c|c|c|}
\hline \multicolumn{3}{|c|}{ Acciones en la aplicación de los PAPsi en la atención de una crisis } \\
\hline & Conveniente - recomendado & Inconveniente - Evitar \\
\hline 1. Contacto & $\begin{array}{l}\text { Escuchar de manera cuidadosa } \\
\text { Refleja sentimientos y hechos } \\
\text { Comunicar aceptación }\end{array}$ & $\begin{array}{l}\text { Contar tu "propia historia" aun. } \\
\text { Ignorar sentimientos o hechos. } \\
\text { Juzgar o tomar partido. }\end{array}$ \\
\hline $\begin{array}{l}\text { 2. Dimensiones del } \\
\text { problema }\end{array}$ & $\begin{array}{l}\text { Plantear preguntas abiertas } \\
\text { Pedir a la persona que sea concreta } \\
\text { Evaluar la mortalidad }\end{array}$ & $\begin{array}{l}\text { Depender de las preguntas sí/no. Permitir } \\
\text { abstracciones continuas. Soslayar las señales de } \\
\text { "peligro". }\end{array}$ \\
\hline 3. Posibles soluciones & $\begin{array}{l}\text { Alentar la lluvia de ideas } \\
\text { Trabajar de manera directa por bloques } \\
\text { Establecer prioridades }\end{array}$ & $\begin{array}{l}\text { Permitir la visión de pasar por un túnel. } \\
\text { Dejar obstáculos sin examinar. } \\
\text { Olear una mezcolanza de necesidades. }\end{array}$ \\
\hline 4. Acción concreta & $\begin{array}{l}\text { Dar un paso cada vez Establecer metas } \\
\text { específicas de corto plazo } \\
\text { Hacer confrontaciones cuando sea necesario } \\
\text { Ser directivo, si, sólo si, debes } \\
\text { Hacerlo }\end{array}$ & $\begin{array}{l}\text { Intentar resolverlo todo ahora. } \\
\text { Realizar decisiones que comprometan por largo } \\
\text { tiempo. } \\
\text { Ser tímido. } \\
\text { Retraerse de tomar decisiones cuando parezca } \\
\text { necesario. }\end{array}$ \\
\hline 5. Seguimiento & $\begin{array}{l}\text { Hacer un convenio para contactar a la } \\
\text { persona atendida } \\
\text { Evaluar los pasos de la acción. }\end{array}$ & $\begin{array}{l}\text { Dejar detalles en el aire, o asumir que el paciente } \\
\text { continuará la acción del plan por sí mismo. } \\
\text { Dejar la evaluación a alguien más. }\end{array}$ \\
\hline
\end{tabular}


Para Cortés y Figueroa (2017) las etapas que se deben cumplir en la aplicación de los Primeros Auxilios Psicológicos son cinco: escucha activa, reentrenamiento de la ventilación, categorización de necesidades, derivación a redes de apoyo, psico-educación. Para cada una de ellas sugieren los siguientes protocolos:

1. Escucha activa: escuche con interés a la persona, entre 10 y 20 minutos. Puede utilizar el texto siguiente para iniciar la conversación:

Mi nombre es __ y hoy estoy aquí para ofrecerle mi ayuda. Primero, y solo si usted desea hablar de ello, vamos a conversar sobre lo que le ha pasado. Luego, si Ud. lo acepta, le enseñaré algunas sencillas técnicas de relajación que le serán muy útiles. Finalmente, veremos en conjunto cómo puede acceder a algunos servicios sociales según sean sus necesidades. ¿De acuerdo, alguna pregunta?... ¿Quisiera contarme algo? (p.28).

2. Reentrenamiento de la ventilación: ofrezca la técnica de la respiración y la relajación, si la persona la acepta, puede abordar la situación de la siguiente manera:

Quizás yo podría ayudarlo(a) a estar un poco más tranquilo(a) luego de lo que Ud. ha vivido con algunos ejercicios de respiración que podría enseñarle. ¿Le gustaría que los practiquemos?... podrá utilizarlos más tarde en su casa si lo desea, lo que podría ayudarlo(a) a estar más tranquilo(a) en los próximos días también (p.28).

3. Categorización de necesidades: identifique y jerarquice las necesidades básicas para obtener seguridad y algún bienestar, por ejemplo necesidad de alimento, agua, abrigo o comunicación con redes de apoyo. El protocolo sugerido es:
Me gustaría saber si quizás hay algo que Ud. esté necesitando en este momento en lo que yo pueda ayudarle... tal vez necesite hablar con su familia, o quizás necesite contactar a [personal de policía], o información respecto a su situación de salud. Intentemos poner en orden lo que necesita para ir paso a paso solucionando los problemas, partiendo primero por lo más urgente, ¿le parece? (p.29).

4. Derivación a redes de apoyo: El personal que va aplicar los PAPsi debe tener recopilada la información de los organismos y grupos de apoyo que en cada caso puedan atender a la persona que está sufriendo una crisis psicológica. Con esta información se puede guiar a la persona con el siguiente protocolo: "OK... ahora que hemos visto algunas de sus necesidades, me gustaría contactarlo con algunos servicios específicos que podrían serle de ayuda..." (p.29).

5.Psicoeducación: Existeun material psicoeducativo que se le debe presentar y explicárselo a la persona, se sugiere hacerlo de la siguiente manera:
Ahora, quisiera entregarle este folleto 'Qué puedo hacer ante una crisis' [pasarle el folleto informativo], el cual contiene información sobre psicoeducación en crisis, además de algunos contactos importantes con instituciones y redes de servicio psicológico y legal que puedan brindarle ayuda si llega a necesitarla a futuro." "Por ejemplo, aquí [señalar] usted podrá encontrar información sobre lo que puede esperar durante los días que vienen... (p.29).

Este material está constituido por información acerca de las emociones y reacciones normales que puede presentar la persona frente a una crisis, se le dan recomendaciones de conductas de afrontamiento positivo. 
En este mismo orden de ideas la Organización Panamericana de la Salud (2002) y Rubin (2000), están de acuerdo en recomendar que se deban evitar las siguientes acciones en el proceso de atender una crisis con la aplicación de PAPsi, estas son:

No aconsejar, se puede sugerir pero no en forma de consejo.

El auxiliador no debe ponerse de ejemplo en alguna situación similar.

No enfatizar excesivamente que hay aspectos positivos de la situación.

Frases como: "Podría haber sido peor" pueden ser contraproducentes.

No minimizar el hecho; la frase "No pasa nada" suele ser negativa.

No bromear, la ironía puede ser ofensiva o contraproducente.

No dramatizar; si la persona llora, el auxiliador no tiene que imitarla.

No engañar a la víctima, ni fingir.

No favorecer la actitud de culparse.

No actuar defensivamente.

No favorecer dependencias directas del afectado con el auxiliador.

Estas recomendaciones deben ser analizadas e interiorizadas por el personal que esté vinculado con la aplicación de los PAPsi, ya que son producto de la práctica de investigadores especializados psicólogos y psiquiatras.

En los centros hospitalarios el personal de enfermería puede enfrentarse a las crisis circunstanciales que se presentan, estos eventos son muy numerosos, y pueden estar referidas a la (a) salud como enfermedades, tratamientos invasivos, amputaciones, enfermedades graves, aborto; (b) muerte inesperada por accidente, enfermedad grave, homicidio o suicidio; (c) actos de violencia.
Por lo tanto el profesional de salud debe estar capacitado para atender no solo la emergencia física y fisiológica sino también la psicológica.

Contrario a todos los hallazgos y recomendaciones de los investigadores y organizaciones reconocidas en los PAPsi para el personal de enfermería, la realidad en los centros de salud de la ciudad de Potosí indica que las atenciones a los pacientes parecen limitarse solo a los aspectos físicos, el tema psicológico parece no ser importante. Esto se explica porque al estar ante un paciente con trauma lo importante es estabilizar y lograr mantener sus signos vitales; tampoco hay mucha disposición en calmar a los familiares, acompañantes o al mismo paciente. Incluso los libros sobre urgencias y emergencias no hacen referencia a la dimensión psicológica (Agustín, 2008; Fernández y Morillo, 2004; Caycedo, 2009). Otro aspecto que se soslaya es la aplicación de estrategias de autocuidado del personal, como parte de los cuidados psicológicos.

Esta investigación se enfoca en los protocolos de aplicación de los PAPsi, en los centros de salud del municipio potosino, porque se considera de suma importancia comprender cuales son los factores que están presentes en el contexto hospitalario y en el personal de enfermería. Asimismo, conocer la disposición que tenga este personal de prestar atención psicológica a las personas que se encuentren viviendo un evento estresor o crisis.

El problema de investigación se plantea con la siguiente interrogante:

¿Cómo es el conocimiento en la intervención de una crisis y Primeros Auxilios Psicológicos del personal de enfermería que se desempeña en los centros de salud del municipio de Potosí en Bolivia?

El objetivo general de la investigación consiste en: Determinar el conocimiento en la intervención de una crisis y Primeros Auxilios Psicológicos del personal de enfermería que se desempeña en los centros de salud del municipio de Potosí en Bolivia. 
Además, de Identificar si el personal de enfermería recibió información sobre intervención en crisis y PAPsi, la fuente por la que recibió. Para de esta manera caracterizar el conocimiento teórico práctico que tiene el personal de enfermería sobre la Intervención en Crisis y los Primeros Auxilios Psicológicos. Logrando con ello establecer los factores de riesgo asociados al conocimiento y a la práctica de las Intervenciones en Crisis y los PAPsi.

\section{MÉTODO}

Se realizó una investigación descriptiva con un diseño de campo de corte transversal y bajo un enfoque misxto, ya que analizaron datos cualitativos y cuantitativos. La población estuvo constituida por 418 profesionales de la enfermería, que se desempeñan en los centros de salud del municipio de Potosí. La muestra estuvo constituida por 171 profesionales, la cual se obtuvo considerando una proporción esperada al $25 \%$ y con un nivel de confianza al $95 \%$ con un p-valor de $5 \%$. Se excluyeron a aquellos profesionales que se encontraban en situación de contrato, por su carácter temporal menor a tres meses, en todo el municipio de Potosí. También se entrevistaron a seis profesionales con cargo directivo, tres médicos y tres profesionales de la enfermería.

En la Tabla 2 se discriminan algunas características de la muestra en cuanto al sexo, edad, nivel del centro de salud en el cual se desempeñan y estudios de postgrado.

Tabla 2. Características de la muestra.

\begin{tabular}{cccc}
\hline Sexo $(F)$ & 8 masculino & 163 femenino & \\
$(\%)$ & 4,7 & 95,3 & 39 tercer nivel \\
Centro de salud (F) & 57 primer nivel & 75 segundo nivel & 22,8 \\
$(\%)$ & 33,3 & 43,9 & \\
Edad (años) (F) & 107 entre 20 y 39 & 64 entre 40 y 59 & 34 maestría \\
$(\%)$ & 62,6 & 37,4 & 19,9 \\
Estudios postgrado (F) & 25 diplomado & & \\
(\%) & 14,5 & 7,6 \\
\hline
\end{tabular}


Las técnicas de recolección de los datos fueron la encuesta y la entrevista. Los instrumentos respectivamente un cuestionario estructurado para identificar el grado de conocimiento y la práctica de los Primeros Auxilios Psicológicos; y una guía de entrevista, de cuatro preguntas abiertas, dirigida al personal directivo de los centros de salud y al personal jefe de enfermería.

En cuanto a la operacionalización de las variables se dimensionan de acuerdo a las características del objeto de estudio (Ver Tabla 3).

Tabla 3. Operacionalización de las variables.

\begin{tabular}{llll}
\hline & & Tipo & Categoría \\
\hline $\begin{array}{l}\text { Características } \\
\text { de la población }\end{array}$ & Edad & Cuantitativa continua & $\begin{array}{l}20 \text { a } 39 \text { anos } \\
40 \text { a } 59 \text { anos } \\
260 \text { anos }\end{array}$ \\
& & & Masculino \\
& Sexo & Cualitativa dicotómica & Femenino \\
& Grado educativo & Ordinal & Pregrado \\
& Nivel del centro de salud & Cualitativa Politómica & Postgrado \\
& $\begin{array}{l}\text { Información en } \\
\text { intervención en crisis }\end{array}$ & Cualitativa Dicotómica politómica & \\
Información en & & \\
intervención en PAPsi & Cualitativa Dicotómica politómica & \\
& Fuente de información & Cualitativa Dicotómica politómica & \\
\hline
\end{tabular}

\section{RESULTADOS}

Con respecto a la existencia de un protocolo de acciones para atender las emergencias psicológicas (Ver Tabla 4).

Tabla 4. Protocolo para la atención de emergencias psicológicas.

El Centro de Salud tiene un protocolo de atención en Emergencias Psicológicas

\section{Frecuencia}

SI

NO
50

121

171
Porcentaje

29,2

70,8

100,0

El 70,8\% de la muestra consideró que en su centro de salud no existe un protocolo para las emergencias psicológicas y si lo hay no lo aplican porque lo desconocen, el $29,2 \%$ responde que si existe en su centro de trabajo un protocolo para atender las emergencias psicológicas. 
Los resultados señalan, en el primer caso, referentes al conocimiento en las intervenciones en crisis, el $52 \%$ responde que si tiene esos conocimientos y el $48 \%$ no los posee. Con respecto a la aplicación de Primeros Auxilios Psicológicos
$48 \%$ si poseen estos conocimientos y $52 \%$ no tienen conocimientos para aplicar los PAPsi.

Al contrastar este resultado con la definición de crisis el 57,3\% escogió la respuesta correcta

Tabla 5. Conocimiento de crisis psicológica y de PAPsi.

\begin{tabular}{lccccc}
$\begin{array}{l}\text { Conocimiento } \\
\text { Psicológica }\end{array}$ & sobre la Intervenciones en Crisis & \multicolumn{2}{c}{$\begin{array}{l}\text { Conocimiento sobre la intervenciones en primeros } \\
\text { auxilios psicológicos }\end{array}$} \\
\hline \multirow{3}{*}{ Válidos } & & Frecuencia & Porcentaje & Frecuencia & Porcentaje \\
& SI & 89 & 52,0 & 82 & 48,0 \\
& NO & 82 & 48,0 & 89 & 52,0 \\
& Total & $\mathbf{1 7 1}$ & $\mathbf{1 0 0 , 0}$ & $\mathbf{1 7 1}$ & $\mathbf{1 0 0 , 0}$ \\
\hline
\end{tabular}

Al contrastar este resultado con la definición de crisis el 57,3\% escogió la respuesta correcta de la definición: "corresponde a una reacción conductual, emocional, cognitiva y biológica de una persona ante un evento precipitante, que se constituye en un estado temporal de trastorno, desorganización y de necesidad de ayuda. Y el $42,7 \%$ estuvo equivocado en la respuesta. (Tabla 6 ).

Tabla 6. Fuente de formación en Emergencia Psicológica.

\begin{tabular}{llcc}
\hline & & Frecuencia & Porcentaje \\
\hline \multirow{4}{*}{ Válidos } & Conferencia & 9 & 5,3 \\
& Taller & 8 & 4,7 \\
& Curso & 33 & 19,3 \\
& Postgrado & 3 & 1,8 \\
& Fuente Informal & 40 & 23,4 \\
& Años de Experiencia & 67 & 39,2 \\
& Formación Autodidacta & 11 & 6,4 \\
& Total & $\mathbf{1 7 1}$ & $\mathbf{1 0 0 , 0}$ \\
\hline
\end{tabular}

En cuanto a la fuente con la cual se formaron en emergencia psicológica, con los años de experiencia el $39,2 \%$, fuente informal $23,4 \%$, y otro porcentaje lo había realizado de forma autodidacta $6,4 \%$. Estos datos son de orden informal con una sumatoria de $69 \%$. La fuente formal por cursos, talleres, conferencias o postgrado corresponde al $31 \%$. El personal de enfermería no tiene una formación estructurada acerca del abordaje de la emergencia psicológica. Los conocimientos del personal de enfermería se encuentran incompletos en cuanto a la prestación de los PAPsi, aspecto que como expresó el responsable de la Red de Servicios de Salud, no debiera ser un conocimiento ajeno sino propio del profesional en enfermería (Fuertes, 2017). 
Cuadro 7. Conocimiento de la primera acción de los PAPsi ante una crisis.

\begin{tabular}{llcc}
\hline & & Frecuencia & Porcentaje \\
\hline \multirow{4}{*}{ Conferencia } & 9 & 5,3 \\
Taller & 8 & 4,7 \\
& Curso & 33 & 19,3 \\
& Postidos & 3 & 1,8 \\
& Fuente Informal & 40 & 23,4 \\
& Años de Experiencia & 67 & 39,2 \\
& Formación Autodidacta & 11 & 6,4 \\
& Total & $\mathbf{1 7 1}$ & $\mathbf{1 0 0 , 0}$ \\
\hline
\end{tabular}

La respuesta correcta ante una situación de crisis psicológica y que requiere la prestación, como primer paso, de los PAPsi es: Establecer un clima mínimo de confianza que favorezca conversación (Proceso de Bienestar Estudiantil (2014) de las respuestas obtuvieron un $48 \%$ de forma correcta y un $37,4 \%$ que no dieron respuestas favorables. Señalando que las profesionales enfermeras abordan, en la mayoría de las situaciones, de forma correcta.

Cuadro 8. Conocimiento de la segunda acción de los PAPsi ante una crisis.

\begin{tabular}{|c|c|c|c|}
\hline & & Frecuencia & Porcentaje \\
\hline & $\begin{array}{l}\text { Análisis de posibles soluciones para que la persona tome } \\
\text { decisiones y actué en búsqueda de solución }\end{array}$ & 20 & 11,7 \\
\hline \multirow[t]{3}{*}{ Válidos } & Comprender las características del problema & 76 & 44,4 \\
\hline & $\begin{array}{l}\text { Establecer un clima mínimo de confianza que favorezca } \\
\text { conversación }\end{array}$ & 34 & 19,9 \\
\hline & Total & 130 & 76,0 \\
\hline Perdidos & Sistema & 41 & 24,0 \\
\hline Total & & 171 & 100,0 \\
\hline
\end{tabular}

La respuesta correcta acerca de la segunda acción ante una situación de crisis psicológica y que requiere la prestación de PAPsi es: comprender de forma más clara las características del problema. El $44 \%$ obtuvo la respuesta correcta. 
Cuadro 9. Conocimiento de la tercera acción de los PAPsi ante una crisis.

\begin{tabular}{llcc}
\hline & & Frecuencia & Porcentaje \\
\hline & $\begin{array}{l}\text { Análisis de posibles soluciones para que la persona tome } \\
\text { decisiones y actué en búsqueda de solución }\end{array}$ & 84 & 49,1 \\
Válidos & Comprender las característica del problema & & \\
& Establecer un clima mínimo de confianza que & 23 & 13,5 \\
& favorezca conversación & 20 & 11,7 \\
& Remitir el caso & 3 & 1,8 \\
& Total & 130 & 76,0 \\
Perdidos & Sistema & 41 & $\mathbf{2 4 , 0}$ \\
\hline
\end{tabular}

El 49,1\% responde correctamente, ya que la tercera acción es: realizar un análisis de posibles soluciones para que la persona tome decisiones y actúe en búsqueda de solución. Siendo la respuesta favorable en un $49,1 \%$ en contraste con las siguientes alternativas que dieron a respuestas no convenientes.

Cuadro 10. Conocimiento de la cuarta acción de los PAPsi ante una crisis.

\begin{tabular}{llcc}
\hline & & Frecuencia & Porcentaje \\
\hline Válidos & $\begin{array}{l}\text { Realizar un análisis de posibles soluciones para que la } \\
\text { persona tome decisiones y actué en } \\
\text { búsqueda de solución }\end{array}$ & 6 & 3,5 \\
& Remitir el caso & 124 & 72,5 \\
& Total & 130 & 76,0 \\
Perdidos & Sistema & 41 & 24,0 \\
Total & & $\mathbf{1 7 1}$ & $\mathbf{1 0 0 , 0}$ \\
\hline
\end{tabular}

Resultados cualitativos de las entrevistas al personal directivo.

La guía de entrevista contempla los siguientes aspectos.

- El centro de salud tiene un protocolo de atención ante emergencias psicológicas.

- En el municipio, a partir de la coordinación de Red Urbano Potosí, y en los distintos establecimientos de salud de primer, segundo y tercer nivel dispone de protocolos ante emergencias psicológicas.

- Conocimiento sobre las Intervenciones en Crisis Psicológica y los Primeros Auxilios Psicológicos.

- Conocimiento sobre la existencia de capacitación en cuanto a intervención en Crisis Psicológica y los Primeros Auxilios Psicológicos.

Respecto a identificar si el centro de salud cuenta con un protocolo de atención ante 
emergencias psicológicas todos respondieron que no existe un protocolo de atención ante emergencias psicológicas. El responsable de los centros de salud de primer y segundo nivel señaló que "el tema de investigación es novedoso como médico debo atender aspectos referentes a la salud física, también a la salud mental. El personal médico también debería tener preparación al respecto porque en algunas ocasiones es el responsable de informar de malas noticias tanto a los pacientes como a los familiares" (Fuertes, 2017). La jefe de enfermeras del Hospital Daniel Bracamonte señaló: "es un tema desconocido, particularmente recién me estoy enterando de este tema. Pero parece importante conocer de este tema" (Tirado 2017); los seis coincidieron en que no existe un protocolo de atención ante emergencias psicológicas y los Primeros Auxilios Psicológicos.

En el municipio, y por información de la coordinación de Red Urbano Potosí, en los establecimientos de salud de primer, segundo y tercer nivel, tanto el personal de enfermería como como los directores de centros de salud consideran que los PAPsi debieran ser parte de la formación del profesional en salud. Aunque identificaron esta estrategia está dirigida a los pacientes y familiares y no lo consideran para el desarrollo de autocuidado.

No existe formación tanto en cursos, talleres o postgrado para el conocimiento, técnicas y normativas de aplicación de los PAPsi. Señalan la importancia de recibir capacitación y formación en intervenciones ante situaciones de crisis psicológica y primeros auxilios psicológicos, ya que los mismos serían un medio adecuado para optimizar servicios en los diferentes centros de salud.

El personal directivo declara su desconocimiento en la aplicación de los PAPsi en los centros de salud. Si bien es cierto que la aplicación de los Primeros Auxilios Psicológicos está más relacionado con los desastres naturales (OPS, 2006; Ramírez, Mónica y Pérez, 2006) también es necesaria su aplicación en los centros hospitalarios. Comunicar malas noticias a los pacientes o familiares de forma adecuada debería ser una competencia de todo personal de salud.

No existen protocolos para la aplicación de los PAPsi y tampoco existe alguna directriz, a este respecto, por parte del Ministerio de Salud del Estado Plurinacional de Bolivia (Ministerio de Salud).

En síntesis los resultados cualitativos y los cuantitativos muestra que no hay un conocimiento en relación con la comprensión y aplicación de los Primeros Auxilios Psicológicos para la intervención de crisis psicológicas en los centros de salud.

Al revisar la Ley del Ejercicio Médico, ley 3131(República de Bolivia, 2005), en su capítulo V, artículo 12, el cual hace referencia a los deberes del médico, en ninguno de sus 12 incisos hacen mención que el médico deba informar de malas noticias al paciente o a los familiares, aunque se supone que el médico tratante debe realizar esta tarea.

\section{CONCLUSIONES}

Los conocimientos acerca de los PAPsi que posee el personal de enfermería provienen de fuentes informales. La educación formal que han cursado no contempla aspectos teóricos o prácticos para la aplicación de los PAPsi para la atención a las crisis psicológicas.

Los conocimientos acerca de los PAPsi son insuficientes, por esta razón el personal de enfermería no posee la confianza para intervenir de forma segura y efectiva una crisis psicológica.

Es necesaria una capacitación educativa planificada y formal para que el personal de enfermería tenga la capacidad de intervenir con seguridad, confianza y efectividad, una crisis psicológica aplicando los PAPsi. 
Los conocimientos que tiene el personal de enfermería respecto a los PAPsi, no son exactos, consideran que los adquirieron con los años de experiencia o los recibieron de fuente informal.

Se descuida el autocuidado profesional ya que no se manejan las técnicas de afrontamiento para las crisis psicológicas generadas por el estrés laboral.

El personal de enfermería no conoce de las Intervenciones en Crisis Psicológica y los Primeros Auxilios Psicológicos, como consecuencia esto reduce su intervención en situaciones específicas de crisis.

\section{REFERENCIAS}

Agustín, C. (2008) Enfermería en Urgencias [Internet].Vol. I. Habana. Cuba:Editorial Ciencias Médicas. httpmedicablogs.diariomedico. comestvezfiles201105completo-1.pdf

Beltrán, E. (2017) Objetivos de la aplicación de los PAP.pdf https://d396qusza40orc. cloudfront.net/pap/docs-on- demand/ modulo-2/V1_3- Objetivos\%20de\%20la\%20 aplicaci\%C3\%B3n\%20de\%20los\%20PAP.pdf

Caycedo, B.y Offìr K. (2009) Intervención psicológica única multifamiliar en la Unidad de Cuidado Agudo de una clínica psiquiátrica. Rev Cienc Salud.7 (3), 29-40

Cortés, P. y Figueroa, R. (2017). Manual ABCDE para la aplicación de Primeros Auxilios Psicológicos En crisis individuales y colectivas. Pontificia Universidad Católica de Chile Escuela de Medicina, Departamento de Psiquiatría Centro Nacional de Investigación para la Gestión Integrada de Desastres Naturales (CIGIDEN)

Cruz, E. (sf). Objetivos de la aplicación de los PAP. https://d396qusza40orc.cloudfront. net/pap/docs-on-demand/modulo-2/ V1_3- Objetivos\%20de\%20la\%20 aplicaci\%C3\%B3n\%20de\%20los\%20PAP.pdf

Federación Internacional de Sociedades de la Cruz Roja, Media Luna Roja. (2014). Primeros Auxilios Psicológicos http://files.unicef. org/paraguay/spanish/Primeros_auxilios_ sicologicos_IFRC.pdf
Fernández, B., Morillo, J (2004). Atención de enfermería en urgencias y emergencias. Madrid, España: Difusión Avances de Enfermería (DAE, S.L.) https://telemedicinadetampico. files.wordpress.com/2011/05/atencion-deenfermeria-en- urgencias-y-emergencias-edmetas.pdf

Fuertes, O. (2017). Personal de Enfermería. Establecimientos de salud de Primer, Segundo y Tercer nivel. Potosí. Bolivia: Coordinación de Red de Servicios de Salud. 67

Hernández, I., y Gutiérrez, L. (2014) Manual Básico de Primeros Auxilios Psicológicos. Guadalajara. México http://www.cucs.udg.mx/principal/ sites/default/files/Manual\%20Primeros\%20 Auxilios\%2 OPsicol\%C3\%B3gicos_2014.pdf

Méndez, E. (2016). Primeros Auxilios Psicológicos y Resiliencia Frente a Experiencias Extremas. Trabajo Final de Grado Montevideo. Universidad de la República Uruguay, Montevideo.http://sifp1.psico.edu.uy/sites/ default/files/Trabajos\%20finales/\%20Archivos/ tfg-_eugenia_mendez.pdf

Ministerio de Salud. Estado Plurinacional de Bolivia. Libros y Normas - Publicado por INASES. https:// www.minsalud.gob.bo/8-institucional/677normas-publicado-por-inases

National Child Traumatic Stress Network, National Center for PTSD. Primeros Auxilios Psicológicos. Guía de operaciones prácticas (2ª . ed.) Estados Unidos: PFA.Tm http://www.nctsn.org/sites/ default/files/pfa/spanish/1- primeros_auxilios_ book_final_comp_guide.pdf

OMS Organización Mundial de la Salud, War Trauma Foundation, \& Visión Mundial Internacional. (2012). Primera ayuda psicológica: Guía para trabajadores de campo. Ginebra: OMS http://apps.who.int/iris/ bitstream/10665/44837/1/9789243548203_ spa.pdf

OPS Organización Panamericana de la Salud (2002) Protección a la Salud Mental en Situaciones de Desastre o Emergencia

OPS Organización Panamericana de la Salud (2006) Guía práctica de salud mental en desastres. Serie manuales y guías sobre desastres. CAPÍTULO III Principales Problemas psicosociales y principales pautas para su atención. Washington, D.C. http://www. 
bvsde.paho.org/bvsacd/cd56/saludmental/ GuiaSaludMental_cap3

OPS Organización Panamericana de la Salud (2006). Guía práctica de salud mental en desastres. Serie manuales y guías sobre desastres. CAPÍTULO VI Primera ayuda psicológica

Pineda, C., y López-López, W. (2010). Atención Psicológica Pos desastres: Más que un "Guarde la Calma". Una Revisión de los Modelos de las Estrategias de Intervención. Sociedad Chilena de Psicología Clínica. 28(2), 155-60

Proceso de Bienestar Estudiantil (2014). Guía de atención paciente en crisis emocional-Primeros Auxilios Psicológicos Universidad Industrial de Santander. https://www.uis.edu.co/intranet/ calidad/documentos/bienestar_estudiantil/ guias/GBE.80.pdf

Proyecto Esfera (2011). Carta Humanitaria y normas mínimas para la respuesta humanitaria. Ginebra: El Proyecto Esfera. http://www. sphereproject.org

Ramírez L. (2016) Primeros Auxilios Psicológicos.
Intervención en crisis emocional.http:// condepah.org/dr/files/PRIMEROS\% 20 AUXILIOS\%20PSICOLOGICOS\%20(PAP). pdf

República de Bolivia. Ley del ejercicio profesional médico. 3131, 3131 agosto de, 2005

Rodríguez, J., Mónica, Z. y Pérez, R. Guía (2006). Guía práctica de salud mental en desastres. Serie manuales y guías sobre desastres [Internet]. OPS/OMS. Vol. VI Primera Ayuda Psicológica. Washington D.C. Estados Unidos; http://www. bvsde.paho.org/bvsacd/cd56/saludmental/ GuiaSaludMental_cap6

Rubín, B. (2000). Intervención en crisis y respuesta al trauma Teoría-Práctica. Bilbao: Desclee de Brouwei

Slaikeu, K. (1996) Intervención en Crisis. Manual para práctica e investigación. (2 ${ }^{\mathrm{a}}$. ed.). México D.F.: Manual Moderno

Tirado S. (2017). Profesionales en Enfermería del Hospital Daniel Bracamonte 